\title{
FREE STREAMLINE FLOW OVER CURVED TOPOGRAPHY
}

\author{
BY \\ A. C. $\mathrm{KING}^{*}$ (University of Nottingham, U.K.) \\ AND \\ M. I. G. BLOOR (University of Leeds, U.K.)
}

\begin{abstract}
The determination of the free streamline of a jet of ideal fluid flowing past a wall of arbitrary shape is considered. A transformation technique is used to formulate the fluid mechanics problem by relating the deflection of the free surface to the angle made by the wall to the undisturbed jet which is found as a solution of a nonlinear integral equation. Linearized solutions, based upon small elevations or depressions in the wall, and nonlinear numerical solutions to this equation are presented for a variety of wall shapes. Some inadequacies of both this linear theory and shallow water theory are found.
\end{abstract}

1. Introduction. This paper considers the problem of determining the free streamline of a jet of ideal fluid which is flowing steadily over an arbitrarily curved wall. The mathematical importance of this arises from the limitation of the classical hodograph techniques to dealing with flows which have a polygonal wall geometry. The practical applications of this type of flow arise both in the hydraulic engineering of fast flow in a channel or a river where the free streamline flow is a leading order approximation to the true flow and also to the aerodynamic interference of a jet by a symmetrical rigid body placed on the centre line of the jet.

Free streamline flows of an ideal fluid are essentially nonlinear problems due to the constant speed condition on the free surface and until the introduction of the concept of a streamline of discontinuity by Helmholtz [1] and the hodograph method by Kirchhoff [2] very little progress on this type of fluid mechanics problem was made. The hodograph method was further developed and applied to both free streamline and cavitation problems by various authors. The limitations of the hodograph method were quickly recognised and arise from the necessity of transforming a polygon in the hodograph plane onto a half-plane by means of the Schwarz-Christoffel transformation. Unless the polygon is of a very simple shape the transformation cannot be integrated explicitly and no analytic solution to the problem can be calculated. Surveys of the successes of the hodograph method can be found in Von Mises [3], Birkhoff and Zarantonello [4], Gilbarg [5] and Gurevich [6]. The recent widespread

Received April 14, 1989.

${ }^{*}$ Current address: Department of Mathematics, University of Keele, Keele, Staffordshire, U.K.

(C)1990 Brown University 
availability of fast digital computers has given some new impetus to the hodograph method as the numerical integration of the Schwarz-Christoffel transformation even in the most complicated geometries is easily accomplished, e.g., Trefethen [7], and numerical solutions to jet and free streamline problems have recently been obtained by various authors, e.g., Dias and Ekrat [8].

The adaptation of the classical hodograph method to flow around a body with a curved boundary was considered by Levi-Civita [9] who obtained a semi-inverse solution to the separated free streamline flow around a curved body in a fluid of infinite extent. A direct formulation of this problem as a nonlinear integro-differential equation for the angle made by the fluid velocity vector was derived by Villat [10] and, in a slightly modified form, was used by Nekrasov [11] to prove both existence and uniqueness of flows around a small circular arc. Nekrasov also found approximate solutions to the equation for this case. Further approximate solutions for flow around circular and elliptic cylinders were given by Brodetsky [12]; an extension and survey of these methods can be found in Birkhoff and Zarantonello.

A generalization of the Schwarz-Christoffel transformation which will transform a half-plane into a region bounded by both polygonal segments and smooth curves was demonstrated by Bloor [13] who used the transformation in the problem of periodic water waves on a fluid of finite depth. This transformation technique has been applied to free surface flows over a step by King and Bloor [14], and to the determination of the cusped free surface flow due to a submerged source, King and Bloor [15], and in all cases results in an integral or integro-differential formulation of the problem. This paper uses the generalized Schwarz-Christoffel transformation to map the region occupied by the fluid onto a half-plane where a simple complex potential can be written down. The free streamline condition of constant speed and the specification in the $(x, y)$-plane of the shape of the wall geometry result in two coupled integral equations for the tangential angles made by the free surface and the wall. A Fourier transform technique is used to decouple the equations and a new nonlinear integral equation for the tangential angle to the wall is derived. Linearized solutions, based upon small elevations or depressions in the wall, and nonlinear numerical solutions are presented for a variety of wall shapes. Some inaccuracies in both this linear theory and Dressler's shallow water theory [16] are noted although the general trend in the free streamline elevation predicted by linear theory is seen to be adequate.

2. Mathematical formulation. The steady two-dimensional free surface flow of an inviscid, incompressible, and irrotational fluid over an arbitrary wall geometry is now considered. It is assumed that no body force such as gravity is acting. A cartesian coordinate system $(X, Y)$ has its origin at some point within the wall which far upstream is taken to be parallel to the undisturbed jet which has uniform speed $U$ and thickness $h$. The above assumptions allow the introduction of a velocity potential $\Phi$ and stream function $\Psi$ such that the complex potential $W=\Phi+i \Psi$ is analytic in the domain occupied by the fluid. Bernoulli's equation can be applied to the free surface on which pressure is constant to give the fluid speed $q$ as a constant there. The stream function $\Psi$ is chosen to have the value zero on the free surface and hence the value $-U h$ on the wall. 


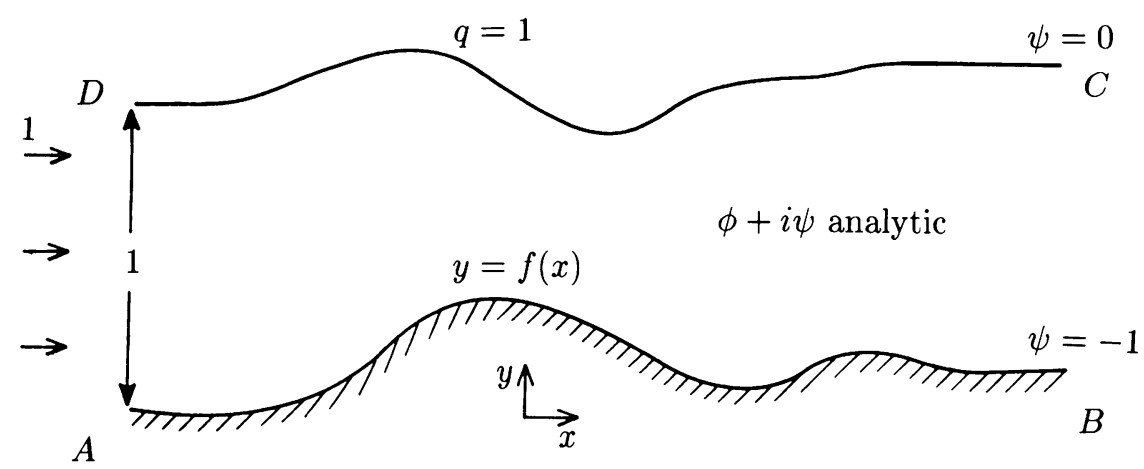

FIG. 2.1 (a). The nondimensional physical plane.

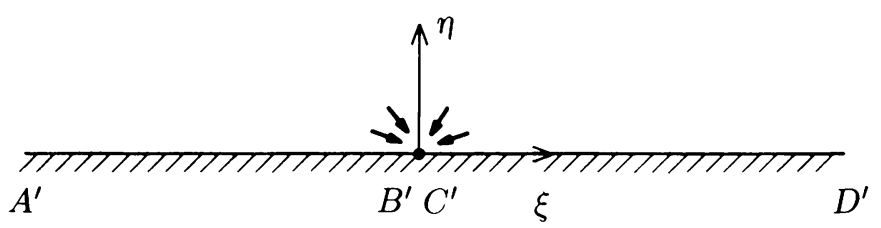

FIG. 2.1 (b). The corresponding transform plane.

This problem is now nondimensionalized using the transformations

$$
x+i y=\frac{X+i Y}{h}, \quad w=\varphi+i \psi=\frac{\Phi+i \Psi}{U h} .
$$

The geometry of this dimensionless flow together with appropriate boundary conditions is shown in Fig. 2.1(a). The process of finding a complex potential for this type of flow in which some of the boundary conditions are to be satisfied on an a priori unknown curve is simplified by transforming the region occupied by the fluid in the physical $z$-plane onto the upper half on a $\zeta=\xi+i \eta$-plane. With the correspondence between the planes shown in Fig. 2.1(a) and (b), the transformation can be written

$$
\frac{d z}{d \zeta}=\frac{-1}{\pi \zeta} \exp \left\{-\frac{1}{\pi} \int_{t=-\infty}^{\infty} \frac{\theta(t)}{\zeta-t} d t\right\}
$$

This transformation is the generalization of the Schwarz-Christoffel formula first considered by Bloor [13] and $\theta(t)$ is the tangential angle to the free surface or wall at the point corresponding to $\zeta=t$. In the $\zeta$-plane the complex potential is that of a sink of strength $1 / \pi$ at the origin so

$$
w(\zeta)=-\frac{1}{\pi} \log \zeta
$$


It is seen from this that on $\eta=0, \varphi=-1 / \pi \log |\xi|$, and $\psi=H(\xi)-1$ where $H$ is the Heaviside step function. The complex velocity can be found from (2.2) and (2.3) as

$$
u-i v=q e^{i \Theta}=\frac{d w}{d z}=\exp \left\{\frac{1}{\pi} \int_{t=-\infty}^{\infty} \frac{\theta(t)}{\zeta-t} d t\right\},
$$

where $\Theta$ is the angle made by the fluid velocity vector at the point corresponding to $\zeta$. As $\zeta$ approaches the real axis the limiting form of $(2.4)$ is

$$
q e^{-i \Theta}=\exp \left\{\frac{1}{\pi} f_{t=-\infty}^{\infty} \frac{\theta(t)}{\xi-t} d t-i \theta\right\},
$$

or

$$
q=\exp \left\{\frac{1}{\pi} f_{t=-\infty}^{\infty} \frac{\theta(t)}{\xi-t} d t\right\}, \quad \Theta=\theta .
$$

Equation $(2.6(\mathrm{~b}))$ shows that on the free surface or the wall the velocity is tangential to the free surface or wall as is required. Application of the free surface condition, $q=1$, gives from $(2.6(\mathrm{a}))$

$$
\frac{1}{\pi} f_{t=-\infty}^{\infty} \frac{\theta(t)}{\xi-t} d t=0, \quad 0<\xi<\infty .
$$

The meaning of Eq. (2.7) is clearer when the contributions which arise from the free surface and wall geometry are shown individually,

$$
\frac{1}{\pi} \int_{t=-\infty}^{0} \frac{\theta(t)}{\xi-t} d t+\frac{1}{\pi} f_{t=0}^{\infty} \frac{\theta(t)}{\xi-t} d t=0, \quad 0<\xi<\infty .
$$

Contributions to the first integral appearing in Eq. (2.8) arise solely from the wall geometry; those in the second integral are the response of the free surface to wall. Equation (2.8) is a singular integral equation of Wiener-Hopf type.

It is convenient at this stage to introduce some new notation and new independent variables which are helpful in the solution of (2.8). Define

$$
\begin{aligned}
& \varphi_{+}=-\frac{1}{\pi} \log \xi, \quad \theta(\xi)=\tilde{\theta}_{+}\left(\varphi_{+}\right), \quad 0<\xi<\infty, \\
& \varphi_{-}=-\frac{1}{\pi} \log (-\xi), \quad \theta(\xi)=\tilde{\theta}_{-}\left(\varphi_{-}\right), \quad-\infty<\xi<0,
\end{aligned}
$$

so that the subscripts \pm on the potential and on $\theta$ show clearly whether they arise from the free surface (which maps into the positive real axis) or the wall (which maps into the negative real axis). Using some elementary transformations, Eq. (2.8) can be rewritten as

$$
\int_{s=-\infty}^{\infty} \frac{\tilde{\theta}_{-}(s) d s}{1+e^{-\pi\left(\varphi_{+}-s\right)}}=f_{s=-\infty}^{\infty} \frac{\tilde{\theta}_{+}(s) d s}{1-e^{-\pi\left(\varphi_{+}-s\right)}}, \quad-\infty<\varphi_{+}<\infty,
$$

i.e.,

$$
\tilde{\theta}_{-} * k_{-}=\tilde{\theta}_{+} * k_{+}
$$

where the standard definition for a convolution product has been used and

$$
k_{ \pm}(\sigma)=\frac{1}{1 \mp e^{-\pi \sigma}} .
$$


Apart from the convenience of recasting (2.8) into a convolution integral equation over the whole of the real line, the change of variables (2.9) has an effect on the transformation (2.2). The limiting form of $(2.2)$ as $\xi$ approaches the real axis is

$$
\frac{d z}{d \xi}=-\frac{1}{\pi \xi} \exp \left\{-\frac{1}{\pi} f_{t=-\infty}^{\infty} \frac{\theta(t) d t}{\xi-t}+i \theta\right\}, \quad-\infty<\xi<\infty .
$$

Introducing $\varphi_{+}$and $\varphi_{-}$on $\xi \gtrless 0$, respectively, and using (2.7) gives

$$
\begin{array}{r}
\frac{d z}{d \varphi_{+}}=\exp \left\{i \tilde{\theta}_{+}\left(\varphi_{+}\right)\right\}, \\
-\infty<\varphi_{+}<\infty,
\end{array}
$$

and

$$
\begin{aligned}
\frac{d z}{d \varphi^{-}}=\exp \left\{\int_{s=-\infty}^{\infty} \frac{\tilde{\theta}_{+}(s) d s}{1+e^{\pi\left(s-\varphi_{-}\right)}}-f_{s=-\infty}^{\infty} \frac{\tilde{\theta}_{-}(s) d s}{1-e^{\pi\left(s-\varphi_{-}\right)}}+i \tilde{\theta}_{-}\left(\varphi_{-}\right)\right\} & , \\
& -\infty<\varphi_{-}<\infty .
\end{aligned}
$$

By taking the real and imaginary parts of (2.12) it is seen that $\tilde{\theta}_{+}$and $\varphi_{+}$are the intrinsic coordinates of the free surface. Equation (2.13) is an equation for the wall shape which involves the unknowns $\tilde{\theta}_{+}, \tilde{\theta}_{-}$.

Taking the Fourier transform of (2.11) and using some standard results

$$
F\left[\tilde{\theta}_{-}\right] \cdot F\left[k_{-}\right]=F\left[\tilde{\theta}_{+}\right] F\left[k_{+}\right] .
$$

The Fourier transforms of $k_{ \pm}$are calculated by contour integration as

$$
F\left[k_{-}\right]=\frac{2 i e^{-k}}{1-e^{-2 k}}, \quad F\left[k_{+}\right]=i\left[\frac{1+e^{-2 k}}{1-e^{-2 k}}\right] .
$$

Equation (2.14) can be rewritten as

$$
F\left[\tilde{\theta}_{+}\right]=F\left[\tilde{\theta}_{-}\right] \operatorname{sech} k .
$$

Using the result that

$$
F\left[\frac{e^{\pi X / 2}}{1+e^{\pi X}}\right]=\operatorname{sech} k,
$$

the solution to Eq. (2.16) can be written, using the convolution theorem for Fourier transforms as

$$
\tilde{\theta}_{+}\left(\varphi_{+}\right)=\frac{1}{2} \int_{s=-\infty}^{\infty} \tilde{\theta}_{-}(s) \operatorname{sech}\left[\frac{\pi}{2}\left(\varphi_{+}-s\right)\right] d s .
$$

Equation (2.17) gives the relation between free surface and wall shape in an explicit form. However a Cartesian equation for the wall shape does not specify $\tilde{\theta}_{-}=\tilde{\theta}_{-}\left(\varphi_{-}\right)$ but specifies $\tilde{\theta}_{-}=\tilde{\theta}_{-}(x)$. To complete the specification of this problem we require a relationship giving $x=x\left(\varphi_{-}\right)$.

Substituting (2.17) into (2.13) and interchanging the order of the resulting double integral gives

$$
\frac{d z}{d \varphi_{-}}=\exp \left\{f_{s=-\infty}^{\infty} \frac{e^{(\pi / 2)\left(s-\varphi_{-}\right)} \tilde{\theta}_{-}(s) d s}{e^{\pi\left(s-\varphi_{-}\right)}-1}+i \tilde{\theta}_{-}\left(\varphi_{-}\right)\right\}
$$


Division of the imaginary and real parts of (2.18) gives

$$
\tilde{\theta}_{-}=\tan ^{-1}\left\{f^{\prime}(x)\right\}
$$

where $y=f(x)$ is the equation of the wall. Integration of the real part of (2.18) and application of the condition that $x \sim \varphi_{-}$as $\varphi_{-} \rightarrow-\infty$ gives

$$
x\left(\varphi_{-}\right)=\varphi_{-}+\int_{t=-\infty}^{\varphi_{-}}\left\{e^{P(t)} \cos \tilde{\theta}_{-}(t)-1\right\} d t,
$$

where

$$
P(t)=f_{s=-\infty}^{\infty} \frac{e^{(\pi / 2)(s-t)} \tilde{\theta}_{-}(s) d s}{e^{\pi(s-t)}-1} .
$$

Equations (2.18), (2.19), (2.20) can be combined to give

$$
\tilde{\theta}_{-}\left(\varphi_{-}\right)=\tan ^{-1}\left\{f^{\prime}\left(\varphi_{-}+\int_{t=-\infty}^{\varphi_{-}}\left\{e^{P(t)} \cos \tilde{\theta}_{-}(t)-1\right\} d t\right)\right\} .
$$

Equation (2.22) is a nonlinear integral equation for $\tilde{\theta}_{-}$over the range $-\infty<\varphi_{-}<$ $\infty$. In principle if the equation is solved for $\tilde{\theta}_{-}$then $\tilde{\theta}_{+}$can be found from (2.17) and then $x\left(\varphi_{+}\right)$and $y\left(\varphi_{+}\right)$from (2.12). In practice it is not possible to find analytic solutions to $(2.22)$ for a curved wall and linearized and numerical nonlinear solutions are considered in the next sections of this paper.

For the case of polygonal wall geometry (2.22) becomes an identity and the solution is written in the form

$$
\tilde{\theta}_{-}\left(\varphi_{-}\right)=\sum_{i=1}^{n} \alpha_{i}\left\{H\left(\varphi_{-}-a_{i}\right)-H\left(\varphi_{-}-a_{i+1}\right)\right\},
$$

where $\alpha_{i}$ is the angle by the $i$ th segment of the polygonal wall with the $x$-axis and $a_{i}, a_{i+1}$ are the velocity potential at the beginning and end of the segment. The length $\ell_{i}$ of the $i$ th segment is given by

$$
\ell_{i}=\int_{t=a_{i}}^{a_{i+1}} \prod_{i=1}^{n}\left|\frac{\left(e^{(\pi / 2) a_{i+1}}-e^{\pi t / 2}\right)\left(e^{(\pi / 2) a_{i}}+e^{\pi t / 2}\right)}{\left(e^{(\pi / 2) a_{i+1}}+e^{\pi t / 2}\right)\left(e^{(\pi / 2) a_{i}}-e^{\pi t / 2}\right)}\right|^{\alpha_{i} / \pi} d t .
$$

Equations (2.17) and (2.12) can be used in conjunction with (2.23) to give the free surface as the solution of

$$
\frac{d z}{d \varphi_{+}}=\prod_{i=1}^{n}\left\{\frac{\left(e^{(\pi / 2) a_{i}}+i e^{\pi \varphi_{+} / 2}\right)\left(e^{\pi a_{i+1}}+e^{\pi \varphi_{+}}\right)^{1 / 2}}{\left(e^{(\pi / 2) a_{i+1}}+i e^{\pi \varphi_{+} / 2}\right)\left(e^{\pi a_{i}}+e^{\pi \varphi_{+}}\right)^{1 / 2}}\right\}^{2 \alpha_{i} / \pi}
$$

It is clear from the above that free streamline flows over polygonal wall shapes are a special case of the more general problem addressed by this paper and that the solution of such problems is reduced to a question of integration. This matter is not pursued further since classical hodograph techniques can be used in this special case.

3. Linearized theory. A linear theory for Eq. (2.22) can be constructed by assuming a wall shape of the form $y=\varepsilon f(x), \varepsilon \ll 1$ and $f(x)=O(1)$. If a solution of the form

$$
\tilde{\theta}_{-}\left(\varphi_{-}\right)=\varepsilon \theta^{(1)}\left(\varphi_{-}\right)+O\left(\varepsilon^{2}\right)
$$


is assumed the standard expansions for $\cos \tilde{\theta}$ and $e^{P}$ are used, then at leading order it is found that

$$
\theta^{(1)}\left(\varphi_{-}\right)=f^{\prime}\left(\varphi_{-}\right)
$$

Equation (2.17) then gives an expression for $\tilde{\theta}_{+}\left(\varphi_{+}\right)$as

$$
\tilde{\theta}_{+}\left(\varphi_{+}\right)=\frac{\varepsilon}{2} \int_{s=-\infty}^{\infty} f^{\prime}(s) \operatorname{sech}\left(\frac{\pi}{2}\left(\varphi_{+}-s\right)\right) d s+O\left(\varepsilon^{2}\right) .
$$

Using the fact that $\tilde{\theta}_{+}\left(\varphi_{+}\right)=O(\varepsilon)$ Eq. (2.12) can be expanded for small $\varepsilon$ and integrated to give

$$
\begin{gathered}
x\left(\varphi_{+}\right)=\varphi_{+}+O\left(\varepsilon^{2}\right) \\
y\left(\varphi_{+}\right)=1+\int_{t=-\infty}^{\varphi_{+}} \frac{\varepsilon}{2} \int_{s=-\infty}^{\infty} f^{\prime}(s) \operatorname{sech}\left(\frac{\pi}{2}(t-s)\right) d s d t+O\left(\varepsilon^{2}\right) .
\end{gathered}
$$

An interchange of the order of integration in (3.4(b)) and elimination of the parameter $\varphi_{+}$using $(3.4(\mathrm{a}))$ gives the equation of the free surfaces as

$$
y(x)=1+\frac{2 \varepsilon}{\pi} \int_{s=-\infty}^{\infty} f^{\prime}(s) \tan ^{-1}\left\{e^{(\pi / 2)(x-s)}\right\} d s+O\left(\varepsilon^{2}\right) .
$$

A more convenient form of this is obtained by integration by parts, using $f(-\infty)=0$,

$$
y(x)=1+\frac{\varepsilon}{2} \int_{s=-\infty}^{\infty} f(s) \operatorname{sech}\left(\frac{\pi}{2}(x-s)\right) d s+O\left(\varepsilon^{2}\right) .
$$

As an example of the application of this linear theory consider a wall shape of the form $y=-\frac{3}{4} \operatorname{sech} \pi x$. The integral appearing in (3.6) is evaluated by contour integration and the free surface takes the form

$$
y(x)=1-\frac{3 e^{\pi x / 2}\left(1+e^{\pi x}-2^{1 / 2} e^{\pi x / 2}\right)}{2\left(1+e^{2 \pi x}\right)} .
$$

A comparison of this result with the nonlinear numerically calculated free surface is shown in Fig. 2.5.

4. Numerical method. To obtain numerical solutions to the nonlinear problem it is necessary to work with a form of Eq. (2.22) which does not involve an infinite range of integration. This is achieved by the integration of the real part of (2.18) between the limits of $\left[\varphi_{-}^{*}, \varphi_{-}\right]$instead of $\left[-\infty, \varphi_{-}\right]$where $\varphi_{-}^{*}$ is some arbitrary point far upstream of any disturbance to the wall shape. Application of the asymptotic result that $x\left(\varphi_{-}^{*}\right)=\varphi_{-}^{*}$ gives

$$
\tilde{\theta}_{-}\left(\varphi_{-}\right)=\tan ^{-1}\left\{f^{\prime}\left(\varphi_{-}^{*}+\int_{t=\varphi_{-}^{*}}^{\varphi_{-}} e^{P(t)} \cos \tilde{\theta}_{-}(t) d t\right)\right\} .
$$

A mesh of discrete $\varphi_{-}$values and corresponding $\tilde{\theta}_{-}$values is defined by

$$
\varphi_{-}^{i}=i h, \quad \tilde{\theta}_{-}^{i}=\tilde{\theta}_{-}\left(\varphi_{-}^{i}\right), \quad-N \leq i \leq M .
$$


The integral in (4.1) is discretized by the trapezoidal rule, with error $O\left(h^{2}\right)$, and $\varphi_{-}^{*}$ is chosen to be $\varphi^{-N-1}$

$$
\tilde{\theta}_{-}^{i}=\tan ^{-1}\left\{f^{\prime}\left(\varphi_{-}^{-N-1}+\frac{h}{2} \sum_{j=-N-1}^{i} w^{j} \exp \left\{P\left(t^{j}\right)\right\} \cos \tilde{\theta}^{j}\right)\right\}, \quad-N \leq i \leq M,
$$

where $w^{j}$ are the weights appropriate to a trapezoidal rule and the $t^{j}=\varphi_{-}^{j}$ are a set of integration points. The discretization of the singular integral $P\left(t^{j}\right)$ is complicated by the singularity at $s=t^{j}$ and the infinite range of integration in the integral. The range of integration is truncated to $\left[\varphi_{-}^{-2 N}, \varphi_{-}^{2 M}\right]$, the integrand being exponentially small outside this range. This truncated range is subdivided into three subranges $\left[\varphi_{-}^{-2 N}, \varphi_{-}^{j-1}\right],\left[\varphi_{-}^{j-1}, \varphi_{-}^{j+1}\right]$ and $\left[\varphi_{-}^{j+1}, \varphi_{-}^{2 M}\right]$. In the first and third of these subranges the integrand is regular and is dealt with by a trapezoidal rule; the error is again $O\left(h^{2}\right)$. In the second subrange the integrand is singular and the integral is evaluated in the sense of a Cauchy principal value by using the Taylor expansion of the integrand about the point $s=t^{j}$; terms of $O\left(h^{3}\right)$ and above are neglected to be consistent with the $O\left(h^{2}\right)$ error arising from the trapezoidal rule. The result after a little algebra is

$$
P\left(t^{j}\right)=\frac{h}{2} \sum_{\substack{k=-2 N \\ k \neq j}}^{2 M} \frac{w^{k} e^{(\pi / 2)\left(s^{k}-t^{j}\right)} \tilde{\theta}_{-}^{k}}{e^{\pi\left(s^{k}-t^{j}\right)-1}}+\frac{2 h}{\pi} \tilde{\theta}_{-}^{\prime}\left(t^{j}\right),
$$

where $\tilde{\theta}_{-}^{\prime}$ is the first derivative of $\tilde{\theta}_{-}$. This derivative can be replaced by a central difference approximation which is accurate to $O\left(h^{2}\right)$ to give $P\left(t^{j}\right)$ as a weighted linear combination of $\tilde{\theta}_{-}^{j},-2 N \leq j \leq 2 M$. The values of $\tilde{\theta}_{-}^{j}$ outside the range $-N \leq i \leq M$ are taken to be the linear values given in (3.2). Provided the mesh is large enough and that $f^{\prime}\left(\varphi_{-}\right)$tends to zero rapidly as $\left|\varphi_{-}\right| \rightarrow \infty$ this does not introduce any significant error into the discretization of Eq. (4.1). Equations (4.2) and (4.3) constitute a set of $N+M+1$ equations of nonlinear algebraic form in $N+M+1$ unknowns $\tilde{\theta}_{-}^{i},-N \leq i \leq M$.

Once the values of $\tilde{\theta}_{-}^{i}$ are found the values of $\tilde{\theta}_{+}$on the free surface can be found using a trapezoidally discretized version of (2.17). Using the notations $\varphi_{+}^{i}=$ $i h, \tilde{\theta}_{+}^{i}=\tilde{\theta}_{+}\left(\varphi^{i}\right)$ and truncating the infinite range of integration to $\left[\varphi_{+}^{-2 N}, \varphi_{+}^{2 M}\right]$ gives

$$
\tilde{\theta}_{+}^{i}=\frac{h}{4} \sum_{j=-2 N}^{2 M} w^{j} \tilde{\theta}_{-}^{j} \operatorname{sech}\left(\frac{\pi}{2}(i-j) h\right), \quad-N \leq i \leq N .
$$

The equation of the free surface (2.12) can be written in integrated form as

$$
x^{i}=x^{-N}+\int_{\varphi_{+}^{-*}}^{\varphi_{+}^{i}} \cos \tilde{\theta}_{+} d \varphi_{+}, \quad y^{i}=y^{-N}+\int_{\varphi_{+}^{-\cdot}}^{\varphi_{+}^{\prime}} \sin \tilde{\theta}_{+} d \varphi_{+}
$$


Discretization of these and application of $x^{-N}=-N h, y^{-N}=1$ gives

$$
x^{i}=-N h+\frac{h}{2} \sum_{j=-N}^{i} w^{j} \cos \tilde{\theta}_{+}^{j}, \quad y^{i}=1+\frac{h}{2} \sum_{j=-N}^{i} w^{j} \sin \tilde{\theta}_{+}^{j} .
$$

\begin{tabular}{|l|l|l|}
\hline$N, M$ & $h$ & $y^{M}$ \\
\hline 40 & 0.15 & 1.00257 \\
\hline 80 & 0.075 & 1.00109 \\
\hline 100 & 0.06 & 1.00057 \\
\hline 120 & 0.05 & 1.00042 \\
\hline
\end{tabular}

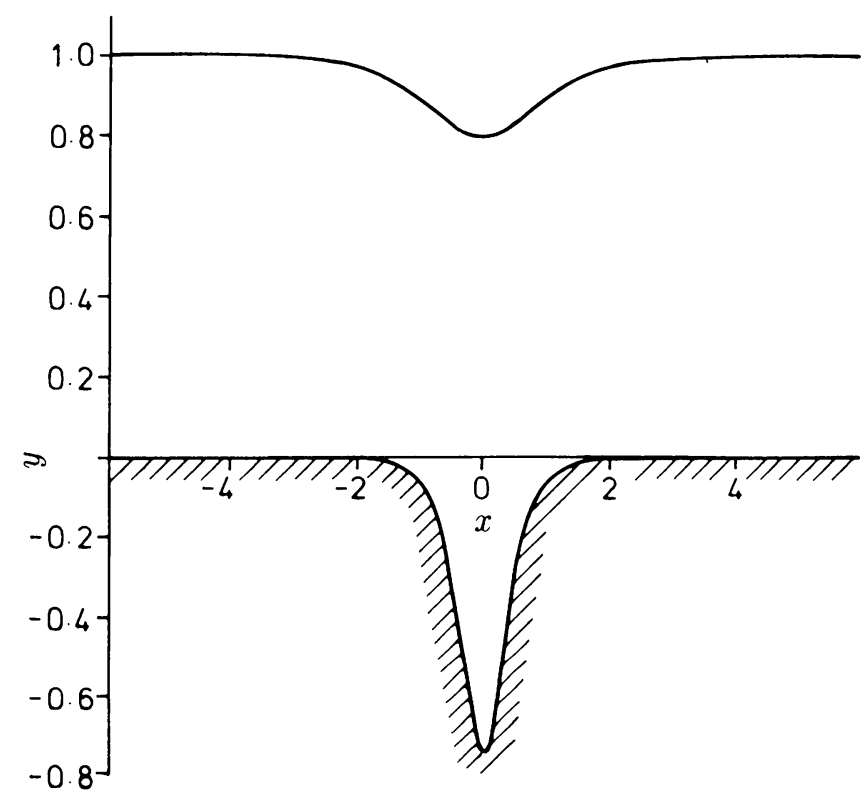

FIG. 2.2. $f(x)=-\frac{3}{4} \operatorname{sech} \pi x$. Inset is convergence of numerical solution with mesh.

The solution of the nonlinear algebraic equations (4.2) and (4.3) was carried out by a hybrid Powell's method. Briefly this is an iterative scheme which solves a system of $n$ equations in $n$ unknowns by correcting the $i$ th iterate by a convex combination of the Newtonian correction and the conjugate gradient correction; a further description of the algorithm can be found in Rabinowitz [17]. This method is available in the N.A.G. library as a FORTRAN 77 subroutine and a program was developed on a DEC VAX 11/785 computer which firstly solved the nonlinear equations and then 
calculated $\tilde{\theta}_{+}^{i}, x^{i}, y^{i}$ from (4.4) and (4.5). The linear solution (3.2) was used as a first approximation to the solution and typically three iterative cycles of the Powell's method were required to produce a converged nonlinear solution. The residual errors in the nonlinear solution were found by substituting the nonlinear solution back into Eq. (4.2) and (4.3) and were less than $10^{-7}$ in norm for all results presented here. Various numerical experiments on mesh extent, mesh fineness and choice of $\varphi_{-}^{*}$ were performed to determine when the solution became mesh independent. It was found that $N=M=100, h=0.06$ and $\varphi_{-}^{*}=\varphi_{-}^{-N-1}$ gave good results for all wall shapes considered. The only direct check on the numerical results is the value of $y^{N}$, the far downstream free surface elevation. A table of the value of $y^{N}$ for a fixed mesh extent but increasing fineness is inset into Fig. 2.2 and shows satisfactory convergence, in view of the $O\left(h^{2}\right)$ discretization error, to the exact value of 1 .

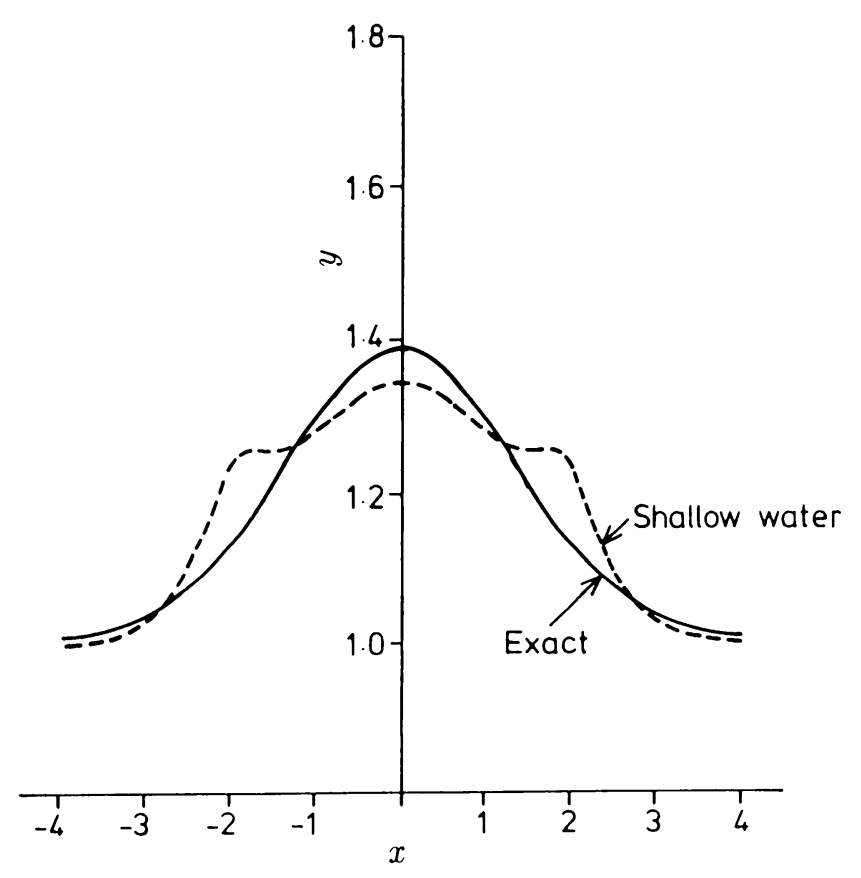

FIG. 2.3. Flow over $f(x)=1 / 2 e^{-1 / 2 x^{2}}$ showing a comparison of the exact theory with shallow water theory.

5. Computed examples and comparison with other work. As there do not seem to be any known analytic or numerical solutions to this problem, three specific examples have been chosen. The change to the program from example to example is straightforward; the use of the mesh parameters above is fixed for simplicity. The number of points could be varied to cater for an unusual example or to give more accuracy 


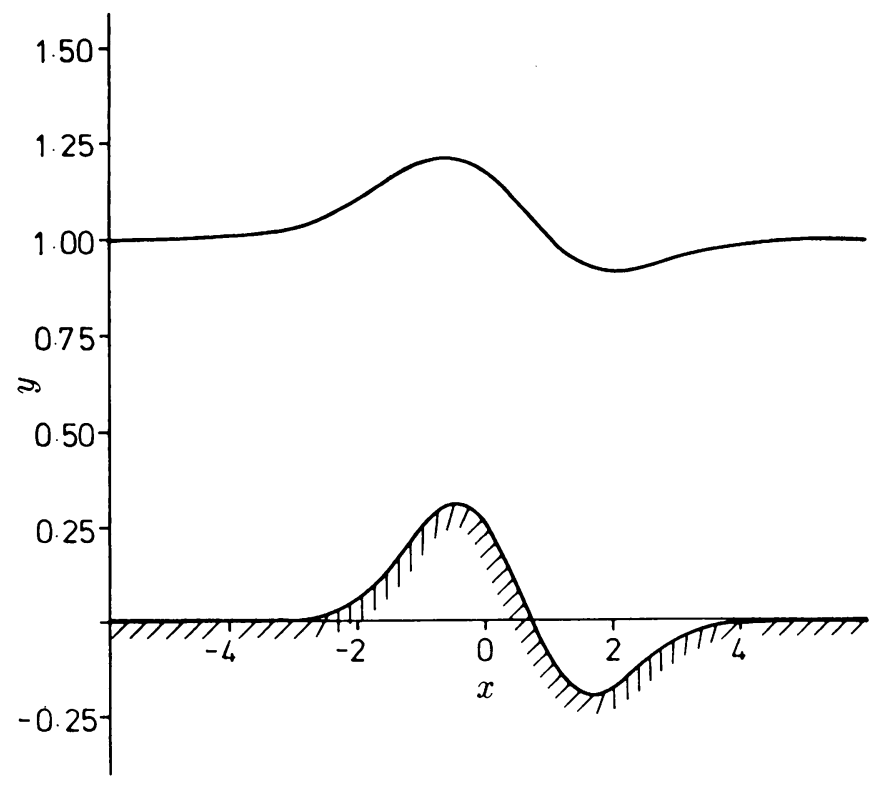

FIG. 2.4. $f(x)=\frac{1}{2} e^{-1 / 2 x^{2}}-\frac{4}{10} e^{-1 / 2(x-1)^{2}}$.

if required. The three examples chosen are

(i) $f(x)=-\frac{3}{4} \operatorname{sech} \pi x$, shown in Fig. 2.2;

(ii) $f(x)=\frac{1}{2} e^{-(1 / 2) x^{2}}$, shown in Fig. 2.3;

(iii) $f(x)=\frac{1}{2} e^{-(1 / 2) x^{2}}-\frac{4}{10} e^{-(1 / 2)(x-1)^{2}}$, shown in Fig. 2.4.

Examples (i) and (ii) are symmetric about $x=0$. The free surface also shows a symmetric fall or rise around $x=0$ as would be expected. The two-dimensional displaced nature of the fluid velocity can be inferred by noting that the elevation of the free surface at $x=0$ is different from the elevation of the wall at this point. A comparison between the linear solution and nonlinear solution, shown in Fig. 2.5, for $f(x)$ as in example (i) shows the inadequacy of a linear theory for Eq. (2.22), although it is seen that the linear result does give a qualitative indication of the true shape of the free surface. Example (iii) is not symmetric about $x=0$ and is included to emphasize that the above analysis and methodology is valid for arbitrary wall shapes.

Finally it is worthwhile comparing the results of this exact theory with a shallow water approximation. Dressler's [16] equations for shallow water flow over a curved topography in the presence of a gravitational body force are

$$
\begin{gathered}
\frac{\partial C}{\partial t}+g \frac{\partial E}{\partial S}=-g S_{f}, \\
\frac{\partial N}{\partial t}+\frac{1}{(1-\kappa N) B} \frac{\partial Q}{\partial s}=0,
\end{gathered}
$$




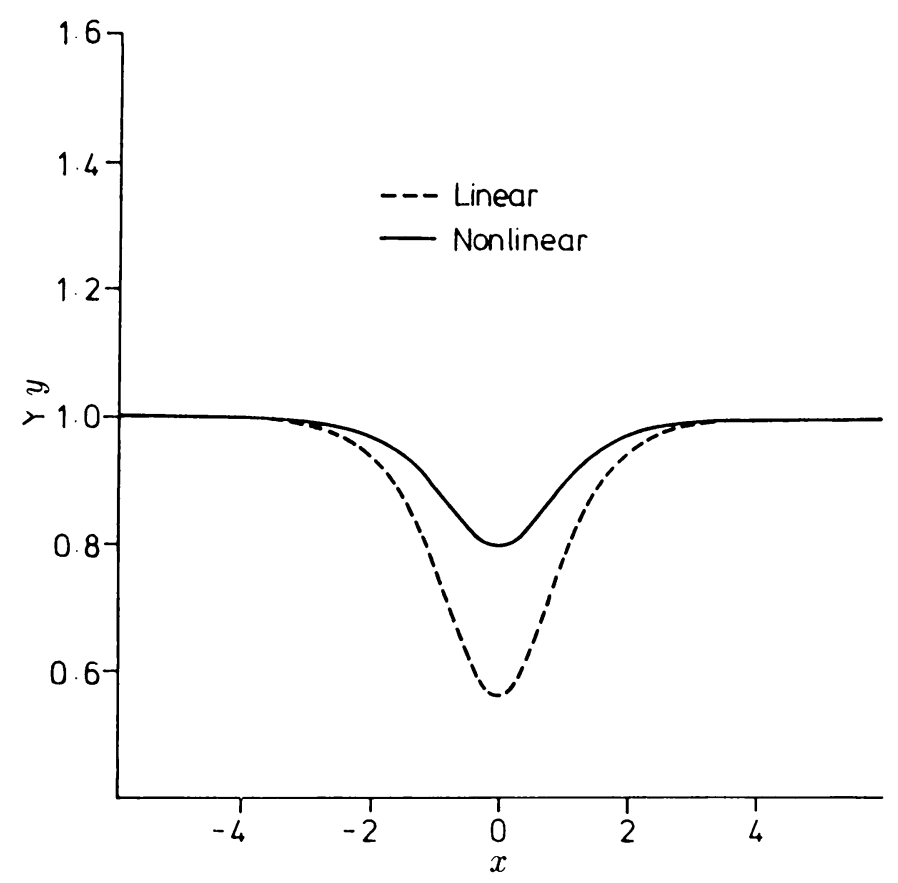

Fig. 2.5. Comparison of linear and nonlinear solutions for $f(x)=-\frac{3}{4} \operatorname{sech} \pi x$.

where

$$
\begin{gathered}
E=\zeta+N \cos \Theta+\frac{p(N)}{\rho g}+\frac{C^{2}}{2 g(1-\kappa N)^{2}}, \\
Q=-\frac{B C}{\kappa} \log _{e}(1-\kappa N) .
\end{gathered}
$$

The symbols $\zeta, B, E, C, N, Q, S_{f}$, and $\kappa$ in these equations represent bed elevation, bed width, energy, speed, free surface elevation, flux, bottom friction, and curvature in a coordinate system based on arclength $(s)$ and normal $(n)$.

For steady flows in which there is no bottom friction, gravitational body force, or atmospheric pressure $(p(N))$ these equations can be reduced to a single equation of the form

$$
(1-\kappa N)+\frac{h \kappa}{\log _{e}(1-\kappa N)}=0
$$

where the conditions $C(-\infty)=U, N(-\infty)=h$, and $\kappa(-\infty)=0$ have been applied upon integration of the Dressler equations. This algebraic equation relates bed curvature to free surface elevation in a direction normal to the bed and can be solved by a simple Newton-Raphson method. A comparison of the Dressler theory with exact numerical solutions is shown in Fig. 2.3 for a bed topography of the form $f(x)=\frac{1}{2} \exp \left(-\frac{1}{2} x^{2}\right)$ and indicates that the shallow water theory is accurate only in 
regions of low bed curvature which correspond to regions where the elevation of the bed is small.

6. Conclusion. A generalization of the Schwarz-Christoffel transformation has been used to formulate the problem of free streamline jet flow over curved wall as a pair of coupled integral equations for the tangential angles onto the free surface and the wall shape. The linear integral equation that arises on the free surface is solved explicitly by the use of Fourier integral transforms and this solution is used to derive a nonlinear integral equation for the angle made by the wall to the undisturbed jet. Linearized and exact nonlinear numerical solutions to this equation are considered and presented for three different wall shapes. The accuracy of the numerical solutions is investigated and is found to be consistent with a known special value of the solution. The inadequacy of the linearized solutions is clearly shown when contrasted with a numerical solution in one of the examples.

\section{REFERENCES}

[1] H. Helmholtz, Uber diskontinvierliche Flüssigkeitsstrahlen, Mber. Akad. Wiss. Berlin, 215 (1868)

[2] G. Kirchhoff, Zur Theorie Freier Flüssigkeitsstrahlen, J. reine angew Math. 70, 289 (1869)

[3] R. von Mises, Selected Papers of Richard von Mises, Amer. Math. Soc., 1963

[4] G. Birkhoff and E. H. Zarantonello, Jets, Wakes and Cavities, Academic Press, New York, 1957

[5] D. Gilbarg, Jets and Cavities, Handbuch der Physik, Vol. 9, Springer, 1960, p. 311

[6] M. I. Gurevich, Theory of Jets in an Ideal Fluid, Academic Press, New York, 1965

[7] L. N. Trefethen, Numerical computation of the Schwarz-Christoffel transformation, SIAM J. Sci. Stat. Comp. 1, 82 (1980)

[8] F. Dias, A. R. Ekrat, and L. N. Trefethen, Ideal Jet Flow in Two Dimensions, J. Fluid Mech. 185, 275 (1987)

[9] T. Levi-Civita, Scie e leggi de resistenza, Rend. Circ. Mat. Palermo 18, 1 (1907)

[10] H. Villat, Sur la résistance des fluides, Ann. Sci. Ec. Norm. Sup. 28, 203 (1911)

[11] N. A. Nekrasov, Sur la mouvement discontinu à deux dimensions de fluid autour d'un obstacle en forme d'arc de cercle, Publ. Inst. Polytech. Ivanovo-Voszniesiensk 32 (1922)

[12] S. Brodetsky, Discontinuous fluid motion past circular and elliptic cylinders, Proc. Roy. Soc. A 102, $361(1923)$

[13] M. I. G. Bloor, Large amplitude surface waves, Pt. 1, J. Fluid Mech. 84, 167 (1978)

[14] A. C. King and M. I. G. Bloor, Free surface flow over a step, J. Fluid Mech. 182, 193 (1987)

[15] A. C. King and M. I. G. Bloor, Cusped free surface flow due to a submerged source, J. Aus. Math. Soc. B 30, 147 (1988)

[16] R. F. Dressler, A new approach to flows over a curved bed, J. Hydraulic Res. 16, 205 (1978)

[17] P. Rabinowitz, Numerical Methods for Nonlinear Algebraic Equations, Gordon \& Breach, 1970 